\title{
MATERNAL AND INFANT HEALTH IS PROTECTED BY ANTIRETROVIRAL DRUG STRATEGIES THAT PRESERVE BREASTFEEDING BY HIV-POSITIVE WOMEN
}

\author{
Louise Kuhn, $P h D$ \\ Gertrude H Sergievsky Center, College of Physicians and Surgeons; and Department of Epidemiology, Mailman School of Public \\ Health, Columbia University, New York, USA
}

The South African Department of Health is justified in withdrawing support for free infant formula. By so doing, it recognises that any intervention that might detract from breast feeding poses a serious threat to infant survival. Since evidence is now strong that antiretroviral drugs used during lactation prevent transmission of infection from a seropositive mother, strategies that promote breastfeeding can now be recommended for enhancing the health of mothers and infants.

The Tshwane Declaration of Support for Breastfeeding in South Africa was recently championed by the national Department of Health as a concrete step to improving maternal and child health in the country. Saloojee, Gray and McIntyre (in the December 2011 edition of this journal) state they are not opposed to this declaration, and welcome the greater support for application of baby-friendly principles in the health services, strengthening community-based programmes to support breastfeeding, and stricter legislation to protect the rights of breastfeeding mothers. They objected to only one item concerning the withdrawal of free formula for HIV-positive women, and lamented that there has been hardly any response from clinicians, health professionals or civil society groups to this decision. Aside from their objections, the overwhelming response to the Tshwane Declaration from clinicians, health professionals and civil society groups has been enthusiastic support. Moreover, the Tshwane Declaration itself was a culmination of more than two years of consultation between the Department of Health and clinicians, health professionals, civil society groups, including activists and women living with HIV, and Saloojee et al. themselves.

The latter authors state that the evidence base for withdrawal of formula is inadequate. In this paper, I present the extensive evidence base supporting the new South African government policies. The evidence is strong that provision of free infant formula is dangerous and that antiretroviral drugs (ARVs) work. I also discuss whether withdrawal of free formula could be considered unconstitutional - a very important accusation, and one which requires thoughtful consideration.

\section{BREASTFEEDING SAVES LIVES}

Saloojee et al. assert that to withdraw support for free formula is a luxury that South Africa can ill afford unless there is 'substantial evidence that the strategy is either ineffective or results in major harm' [emphasis added]. While these are reasonable criteria on which to make any decision about public health, it is extraordinary that they appear to disregard the overwhelming evidence from around the African continent, including countries in southern Africa, that formula feeding is associated with significantly higher mortality in young infants. It is precisely the accumulation of substantial evidence that provision of infant formula is either futile or results in major harm that informed the international recommendations released by the World Health Organization to guide national ministries of health.'

Human breast milk is exquisitely regulated, containing not only nutrients but also immunologically active components to protect newborns against disease and support the maturation of their own immune system. ${ }^{2}$ Medical research dating back to the Middle Ages identified that orphans and abandoned infants would die unless human breast milk were provided. ${ }^{3}$ An experiment was undertaken in the 1970s by formula manufacturers, confident in their 'modern' product, who began marketing it in African countries. Provision of infant formula correlated with infant deaths. ${ }^{4}$ Fortunately, these deaths also sparked effective pro-breastfeeding advocacy that has helped to shape global public health initiatives. There are extensive biological, clinical, epidemiological and programmatic data indicating that infant formula results in major harm to infants and their mothers. Consequently, it is a falsehood to say that evidence showing the major harm associated with provision of formula is 'simply lacking'. There is overwhelming evidence of the harmful effects of formula feeding in the general population in southern Africa and elsewhere for decades. Until recently, there was indeed a lack of evidence of any comparable effect among HIV-infected mothers and their exposed but uninfected infants. Yet there is now substantial evidence in better- and less-resourced settings, including the better-resourced settings of South Africa and Botswana, that formula feeding results in elevated death rates among children who would otherwise be HIV-uninfected and alive. The serious threat to infant survival is the most important justification for the withdrawal of Department of Health support for infant formula. 


\section{IS HIV IN SOUTH AFRICA THE EXCEPTION?}

Saloojee et al. do not appear to be aware of this expanse of biological, clinical, epidemiological and programatic research. It seems that their position can only be held if they subscribe to two types of 'exceptionalism': (i) HIV exceptionalism and (ii) South African exceptionalism.

Postnatal transmission of HIV through breastfeeding is indeed a special case that requires cautious and courageous consideration of appropriate infant feeding policy. HIV transmission can occur throughout the period of breastfeeding, therefore complete abstention from breastfeeding will obviously not permit any transmission to occur via this route. Abstention from breastfeeding will not, however, prevent intrauterine or intrapartum transmission. In the absence of interventions, most ( 70\%) infants born to and breastfed by HIV-positive mothers will remain uninfected. When HIV-positive women avoid breastfeeding with the goal of preventing the proportion of vertical transmission attributable to breastfeeding, they place their infants at risk of malnutrition, pneumonia and diarrhoeal morbidity and mortality as well as increasing the child's risk of developmental and cognitive delays. This is the nub of the dilemma, and provision of free formula is not a solution; instead, it's part of the dilemma that the HIV epidemic has made us face.

In the era prior to the demonstration that ARVs used during lactation can provide a constructive solution to the infant-feeding dilemma, two wish-fulfillment strategies were used instead: Either deny that HIV is transmitted through breastfeeding or deny that there are substantial risks of death and other serious outcomes from formula feeding. Study after study clearly showed that HIV is transmitted to infants through breastfeeding. Denial of the dangers of formula became the more popular position. In 2000, the WHO recommended that HIV-infected women provide formula feeds to their infants as a means of preventing HIV infection. This guidance was based on the premise and intention that public health programmes could be set up that would eliminate the risks associated with handing out formula feeds. This strategy was very powerful because it was able to mobilise resources to buy formula for programmes and for research. But the strategy set aside the large body of breastfeeding research that had been conducted among non-HIV-infected women that had described and quantified the excess risk of death associated with formula feeds; it called for new research and evidence to record the experiences and measure the effects of using formula feeds by HIV-infected mothers.

For better or worse, several groups, including my own, bought into this notion and conducted studies to test whether complete avoidance of breastfeeding, or shortening the duration of breastfeeding, would have adverse consequences for infants born to HIV-positive mothers. Sadly, they did. These well-conducted, rigorous research studies with results that have been reviewed by peer scientists prior to publication in leading medical journals were conducted in a wide range of settings in Africa, including better-resourced settings such as Botswana and South Africa. For example, in a clinical trial in urban Botswana where women were randomised either to formula from birth or breastfeeding for 6 months, a doubled risk of death was observed among uninfected infants born to HIV-infected mothers. ${ }^{5}$ In this study, participants were carefully screened to ensure all had access to clean water and adequate sanitation, formula was provided free, counselling and support around formula feeding was extensive, and there was a well-functioning health service safety net. In another example in a well-resourced area in rural Uganda, with a sophisticated health service, women were counselled about infant feeding options following AFASS (affordable, feasible, acceptable, sustainable and safe - the acronym summarising the criteria that were proposed at that time as the requirement for formula feeding to be the better choice) and a 6 -times greater risk of infant mortality was observed among women who selected formula feeding because they felt it was 'AFASS' for themselves. ${ }^{6}$ There are several other studies, including numerous studies from South Africa. ${ }^{7-14}$ The consistency of the findings across diverse settings, across different study designs and with established biological processes makes it highly unlikely that the dangers of formula can be explained away as part of the vagaries of clinical research methodology. The findings of these studies, in conjunction with research findings demonstrating the efficacy of ARVs to significantly reduce the risk of HIV infection through breastfeeding, iteratively led the WHO to revise its recommendations from a position of recommending formula feeds as the default feeding practice for HIV-infected mothers, to recommending breastfeeding with ARVs.

Saloojee et al. dismiss this large body of research with the claim that it comes from settings with much higher rates of infant morbidity and mortality than those observed in South Africa (or those parts of South Africa with more resources). This is not true i.r.o. Botswana and other countries, such as Zambia and Malawi, that are more economically disadvantaged, and manifests a confusion between an absolute and a relative risk. An absolute risk quantifies the likelihood that an event will occur in a group; e.g. the risk of dying is 40 per 1000 . A relative risk compares two groups: group A has an absolute risk of 40 per 1000 and group $B$ has an absolute risk of 80 per 1000 , therefore the relative risk of group B v. group A is double. Even in countries with very low absolute rates of infant mortality, such as the USA, UK and the Netherlands, formula increases mortality; i.e. the relative risk is elevated. ${ }^{15-17}$ But in countries with higher absolute infant mortality rates, the same relative risks translate into a larger absolute number of infant deaths. Moreover, synergy occurs: in populations with high absolute mortality rates, relative risks of death owing to formula are also higher; for example, water contamination, lack of access to adequate sanitation and poor health service infrastructure exacerbate the dangers of formula. But economic disadvantage does not create the biological disadvantage of formula. There is no threshold below which formula no longer causes harm. Breastfeeding saves lives in all countries - South Africa is no exception.

Saloojee et al. misquote three studies ${ }^{18-20}$ as evidence that replacement feeding can be safely accomplished. These studies do report equivalent or better outcomes with replacement feeding; however, the outcome reported is HIV-free survival, and at a time when ARVs were not available to prevent HIV infection through breastfeeding. HIV-free survival is a composite endpoint defined by the absence of either infant HIV infection or infant death. As a public health indicator, it is useful as it reminds us that there is little point in saving infants from HIV if they are only going to die of other causes. However, consideration of only HIV-free survival does not provide proof of safety of formula feeds. Equivalent HIV-free survival means that the number of HIV infections averted has been cancelled by the number of additional uninfected deaths caused. 


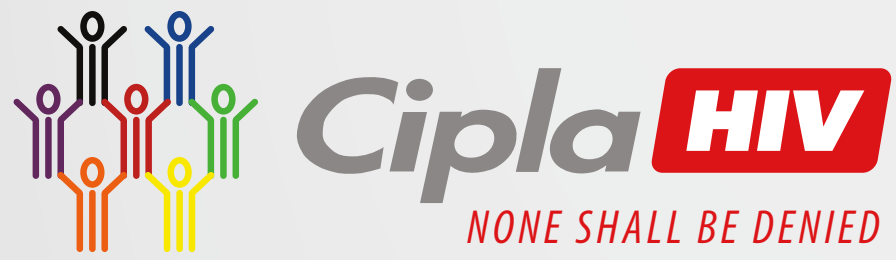

\section{NEW}

\section{FDC}

Tenofovir $300 \mathrm{mg}$

Emtricitabine $200 \mathrm{mg}$

Efavirenz $600 \mathrm{mg}$

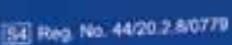

\section{Odimune}

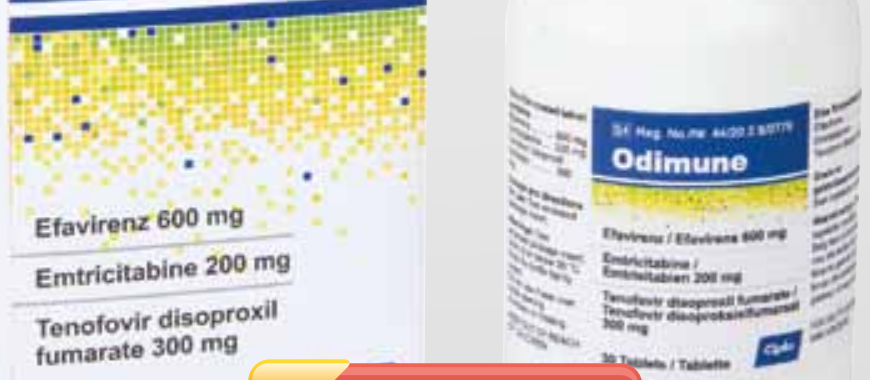

\section{3-in-1 ONCE DAILY}

Recommended as preferred 1st line regimen by national and international guidelines ${ }^{2,3}$

\section{"Simplify, simplify."}

Henry David Thoreau Philosopher 
These stark statistics do not resolve the question of whether breastfeeding alone or formula feeding is the better feeding practice for HIV-exposed infants. New data from research studies undertaken in over 6 countries, including South Africa, completely transform the context in which the dilemma of infant feeding by HIV-infected mothers should be considered. ARV intervention can be used during breastfeeding to reduce the risk of transmission. ${ }^{21-28}$ None of the studies referred to above used ARVs during breastfeeding; the Kenyan study was done before even short-course perinatal interventions became available. These studies are uninformative for the current era when ARVs are available to prevent transmission through breastfeeding. Saloojee and colleagues remain locked into an evidence base and paradigm that does not recognise the potency of $A R V$ s and the opportunity they present to improve the health and survival of HIV-exposed infants.

\section{SOUTH AFRICA HAS PERSISTING INEQUITIES IN HEALTH AND WEALTH}

As an argument in support of free formula, Saloojee et al. remind us that South Africa is not a single homogenous country. This is absolutely true. South Africa is a country is with gross disparities in wealth, health and living conditions. This is not an argument for the government to support formula for the better-off. To the contrary, new government policies can serve to reduce inequities and provide highly effective interventions to everyone and not just a number of favoured groups; the new national policies proactively consider the needs of the poor first - as public health policies should.

In Saloojee et al.'s view, the government should provide free formula for women in the wealthier provinces, such as Gauteng and Western Cape, where women are sufficiently well-off to meet AFASS criteria but not so much as to purchase formula themselves. Yet this contradicts the first ' $A$ ' of AFASS, which is 'affordable' $-\mathrm{a}$ point which they seem to ignore. But it's not actually the A which is most relevant - it's the $S$ for safety. Back to the first point: formula feeding is dangerous.

\section{STUDY THE NUMBERS - THEY MATTER}

It is therefore, reasonable to ask why HIV-positive women in the United States are required to formula feed. It is imperative to pay close attention to the actual absolute and relative risks in the South African context. Saloojee et al. in their protest to the Mail \& Guardian claim that, since infant mortality may be as low as 25 per 1000 in some better-off parts of South Africa, this figure is below the 'accepted' threshold where formula feeding can be considered 'safe'. The basis for this claim is mathematical modelling, conducted by several different groups (including myself) ${ }^{29}$ in the 1990s, calculating the competing risks of HIV transmission associated with breastfeeding $v$. the increased risk of uninfected child deaths owing to abstention from breastfeeding. A 'safe' threshold is the point at which the number of HIV infections averted by formula is exactly equivalent to the number of deaths caused by formula feeding hardly a basis for a resounding endorsement of formula. Nevertheless, the primary limitation of the models used by Saloojee et al. is that they ignore the new opportunities provided by ARV strategies. ARVs, when used throughout lactation, significantly reduce the risk of HIV transmission via lactation. ${ }^{30}$ If one applies the new rates of HIV transmission observed when ARVs are given, the infant mortality rate has to fall to below 10 per 1000 before the increased number of deaths caused by formula feeding is counterbalanced by the number of HIV infections averted. Only when the infant mortality rate is $<8$ per 1000 live births, is formula able to save one child per 1000 . If transmission rates are lower than assumed in the model, and are as

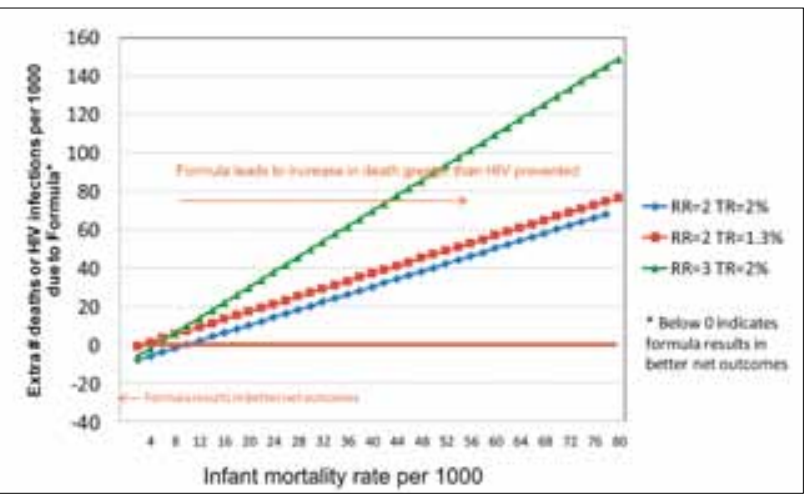

Fig. 1. Breastfeeding with ARVs results in better HIV-free survival when infant mortality rates exceed 10 per 1000 . The graph shows excess adverse outcomes (uninfected infant deaths or HIV infections) per 1000 as a result of formula-feeding, compared with breastfeeding among HIV-infected women in populations of varying infant mortality rates. The models allow a transmission rate (TR) of 2\% assuming ARVs are given and a best-case scenario of $1.3 \%$, consistent with the Botswana clinical trial ${ }^{22}$ of which $0.3 \%$ were due to breastfeeding acquired infections. The model assumes a relative risk (RR) of 2 which is consistent with best-case scenario of clinical trial-supported formula feeding in a better-off environment ${ }^{5}$ and considers $R R=3$ more likely to represent the programmatic setting. All values $>0$ indicate that breastfeeding results in better net outcomes; values $<0$ indicate that formula-feeding results in better net outcomes.

low as observed in clinical trials, such as the trial in Botswana, ${ }^{22}$ or risks associated with formula feeding are higher than observed in clinical trial settings, ${ }^{5}$ as is likely to occur in practice, infant mortality rates need to be even lower before formula can be considered a desirable option (Fig. 1). The infant mortality rate is nowhere near this level in any of the populations affected by HIV in South Africa, even in the wealthier provinces. New government policies take into account the newest up-to-date data, in contrast to the complaints made by Salojee et al. that rely on out-of-date data and arguments that exclude the availability of ARVs.

\section{ANTIRETROVIRAL DRUGS PROVIDE A SOLUTION TO THE INFANT FEEDING DILEMMA}

Many commentators are blithely optimistic about the safety of formula, yet this optimism does not extend to ARV strategies. Regarding the benefits of ARV strategies to prevent mother-tochild HIV transmission, they state these strategies are unproven, based on inference, and with many 'unanswered questions'. This is surprising, since these authors have been at the forefront of testing ARV drug strategies and have published data showing the efficacy and safety of drug interventions and have been highly active in supporting their successful roll-out in Gauteng and elsewhere in South Africa. ${ }^{31,32}$ South African researchers have a stellar record in implementing ARV-based programmes including demonstrating the capacity of the routine health services to provide effective ARV strategies for pregnant HIV-positive women. ${ }^{33-35}$ This is not to say that ARV programmes are easy to implement and that they may fall short. But pessimism, and claiming that failure to implement perfect programmes will have 'drastic consequences', damages mobilisation of resources and the will to implement these programmes. ARV drug strategies are highly effective in reducing mother-to-child HIV transmission through all routes, including breastfeeding, and save women's lives. Programmes to implement these strategies should be supported, not disparaged.

My major concern about this pessimism is that it implies that formula is a better option than ARV drugs. This is deeply 
disturbing because formula does nothing to prevent mother-tochild transmission that can occur during pregnancy and delivery. Formula does nothing to improve maternal health. Even if formula is provided, ARV treatment and prophylactic regimens remain vital. Based on current criteria, a large proportion of pregnant HIVpositive women meet criteria for ARV treatment. They need this treatment as a matter of urgency for their own survival and wellbeing, and this treatment needs to be lifelong. To my mind, it is problematic to argue against ARV therapy because formula is more cost-effective in preventing HIV transmission in a select group of mothers and children. Women who meet criteria for treatment are responsible for a large proportion of the infant infections ( $>80 \%$ of postnatal infections). ${ }^{36}$ Therefore, purely implementing standard adult guidelines for provision of ARV therapy for pregnant women who require it based on their own health status would address the majority of postnatal HIV infections and would also reduce maternal deaths. Choices of infant nevirapine prophylaxis (option A), or therapeutic regimens that are stopped after the cessation of breastfeeding (option B), are available to address the remaining small proportion, but apply only to those asymptomatic women with high CD4 counts. The focus of public health interventions needs to be on reaching the women who need treatment for their own health and who are also most likely to transmit.

\section{HIV IS NOT THE ONLY DISEASE FROM WHICH CHILDREN NEED TO BE PROTECTED}

Saloojee et al. argue that '[a]n HIV-free generation can never be achieved while breastfeeding continues.' This is true. But this statement could be more properly rephrased 'An HIV-free generation can never be achieved while pregnancy continues.' Current ARV drug regimens do not result in zero transmission even in formula-fed populations. When ARV drug regimens were started early in pregnancy and continued through breastfeeding in a study in Botswana, the overall transmission rate, including transmissions that occurred during breastfeeding, was $1.1 \%$. More than $75 \%$ of the HIV infections were detected at birth and had occurred before delivery. Transmission during 6 months of breastfeeding when ARV drugs were given was $0.28 \% .{ }^{22}$ Eliminating breastfeeding will not eliminate HIV transmission. Eliminating breastfeeding will, however, increase infant mortality.

\section{BREASTFEEDING RIGHTS AND WRONGS}

It was not clear from the arguments presented by Saloojee et al. what the basis was for the charge that withdrawal of free formula was unconstitutional. It may be the denial of the 'opportunity to have an HIV-uninfected child' that will result if women are denied access to formula despite meeting AFASS criteria. This rhetoric is seductive but not based on fact. Formula will not guarantee that an HIV-positive woman has an uninfected child. Without ARV drugs, transmission will occur during pregnancy or delivery in about a quarter of women. With adequate ARV drugs given during pregnancy and then stopped, transmission rates would be around $2 \%$, assuming complete abstinence from breastfeeding. A woman may have a 'right' (in the broadest sense of the word) to purchase harmful commodities if she so chooses - just as she has a 'right' to smoke during pregnancy if she so chooses. However, to claim that a woman has a constitutional right to be given harmful products by the health services simply because they prevent HIV transmission, is wrong. Moreover, Saloojee et al. fail to mention children's rights, also protected in the South African constitution and detailed in the Convention of the Rights of Children. This is more than just avoidance of HIV infection.
Health policies should not be decided upon by popularity contest. National health authorities should solicit opinions on policies so that they are sensitive to communities' needs, but the policies themselves need to be based on biological and public health principles and evidence. Involvement of the HIV-infected and -affected community is central. Children, who can be both infected and affected by HIV, need special lobby groups to attend to their interests. The majority of HIV-positive women care about HIV transmission to their infants and their overall health, well-being and survival. The answer of the health service to an HIV-positive woman's question about how best to feed her infant should not be a blunt 'your choice'.

\section{A WAY FORWARD}

It is time to put aside polarising debates and conflicts, and come together to address the fundamental public health challenges facing South Africa. Programmes to support breastfeeding need to be strengthened. This includes addressing the education of healthcare workers so that correct information is conveyed to parents, as well as activism to challenge labour and other policies that deny the rights of breastfeeding women. HIV can be treated with ARVs, and those receiving ARVs have a very low risk of transmitting HIV to their child or sexual partners. We should synergise to ensure that all people living with HIV have access to effective care and treatment. Strengthening these ARV programmes can greatly improve maternal and child health in South Africa.

\section{REFERENCES}

World Health Organization. Guidelines on HIV and infant feeding: Principles and recommendations for infant feeding in the context of HIV and summary of evidence. http://wwwwhoint/maternal child_adolescent/documents/9789241599535/en/ 2010 (accessed 27 January 2012).

2. Labbok MH, Clark D, Goldman AS. Breastfeeding: maintaining an irreplaceable immunological resource. Nat Rev Immunol 2004;4:565-572.

Mathews-Grieco SF. Breastfeeding, wet nursing and infant mortality in Europe (1400-1800). In: Historical perspectives on breastfeeding. United Nations Children's Fund: UNICEF; 1991:15-60. Jelliffe DB, Jelliffe EF. Human Milk in the Modern World. New York: Oxford University Press, 1978. Thior I, Lockman S, Smeaton LM, et al. Breastfeeding plus infant zidovudine prophylaxis for 6 months vs formula feeding plus infant zidovudine for 1 month to reduce mother-to-child HIV transmission in Botswana: a randomized trial: the Mashi Study. JAMA 2006;296:794-805.

6. Kagaayi J, Gray RH, Brahmbhatt $\mathrm{H}$, et al. Survival of infants born to HIV-positive mothers by feeding modality in Rakai, Uganda. PLOS ONE 2008;3:e3877. [http://dx.doi.org/10.1371/journal. pone.0003877].

Kuhn L, Sinkala M, Semrau K, et al. Elevations in mortality due to weaning persist into the second year of life among uninfected children born to HIV-infected mothers. Clin Infect Dis 2010;54:437-444.

8. Taha TE, Hoover DR, Chen S, et al. Effects of cessation of breastfeeding in HIV-1-exposed, uninfected children in Malawi. Clin Infect Dis 2011;53:388-395.

9. Coovadia HM, Rollins NC, Bland RM, et al. Mother-to-child transmission of HIV-1 infection during exclusive breastfeeding in the first 6 months of life: an intervention cohort study. Lancet 2007;369:1107-1116.

10. Doherty T, Chopra M, Jackson D, Goga A, Colvin M, Persson LA. Effectiveness of the WHO/UNICEF guidelines on infant feeding for HIV-positive women: results from a prospective cohort study in South Africa. AIDS 2007;21:1791-1797.

11. Onyango-Makumbi C, Bagenda D, Mwatha A, et al. Early weaning of HIV-exposed uninfected infants and risk of serious gastroenteritis: Findings from two perinatal HIV prevention trials in Kampala, Uganda. J Acquir Immune Defic Syndr 2010;53:20-27.

12. Kafulafula $G$, Hoover DR, Taha TE, et al. Frequency of gastroenteritis and gastroenteritisassociated mortality with early weaning in HIV-1-uninfected children born to HIV-infected women in Malawi. J Acquir Immune Defic Syndr 2010;53:6-13.

13. Creek TL, Kim A, Lu L, et al. Hospitalization and mortality among primarily nonbreastfed children during a large outbreak of diarrhea and malnutrition in Botswana, 2006. J Acquir Immune Defic Syndr 2010;53:14-19.

14. Homsy J, Moore D, Barasa A, et al. Breastfeeding, mother-to-child HIV transmission, and mortality among infants born to HIV-Infected women on highly active antiretroviral therapy in rural Uganda. J Acquir Immune Defic Syndr 2010;53:28-35.

15. Quigley MA, Kelly YJ, Sacker A. Breastfeeding and hospitalization for diarrheal and respiratory infection in the United Kingdom Millennium Cohort Study. Pediatrics 2007;119:e837-e842.

16. Duijts L, Jaddoe VW, Hofman A, Moll HA. Prolonged and exclusive breastfeeding reduces the risk of infectious diseases in infancy. Pediatrics 2010;126:e18-25.

17. Chen A, Rogan WJ. Breastfeeding and the risk of postneonatal death in the United States. Pediatrics 2004;113:e435.

18. Mbori-Ngacha D, Nduati R, John G, et al. Morbidity and mortality in breastfed and formula-fed infants of HIV-1-infected women: A randomized clinical trial. JAMA 2001;286:2413-2420.

19. Leroy V, Ekouevi DK, Becquet $R$, et al. 18-month effectiveness of short-course antiretroviral regimens combined with alternatives to breastfeeding to prevent HIV mother-to-child transmission. PLOS ONE 2008;3:e1645.

20. Franke MF, Stulac SN, Rugira IH, et al. High human immunodeficiency virus-free survival of infants born to human immunodeficiency virus-positive mothers in an integrated program to 
decrease child mortality in rural Rwanda. Pediatr Infect Dis J 2011;30:614-616.

21. Chasela CS, Hudgens MG, Jamieson DJ, et al. Maternal or infant antiretroviral drugs to reduce HIV-1 transmission. N Engl J Med 2010;362:2271-2281.

22. Shapiro RL, Hughes MD, Ogwu A, et al. Antiretroviral regimens in pregnancy and breast-feeding in Botswana. N Engl J Med 2010;362:2282-2294.

23. Taha TE, Kumwenda J, Cole SR, et al. Postnatal HIV-1 transmission after cessation of infant extended antiretroviral prophylaxis and effect of maternal highly active antiretroviral therapy. Infect Dis 2009:200:1490-1497.

24. Tonwe-Gold B, Ekouevi DK, Viho I, et al. Antiretroviral treatment and prevention of peripartum and postnatal HIV transmission in West Africa: evaluation of a two-tiered approach. PLOS Medicine 2007;4:e257.

25. Palombi L, Marazzi MC, Voetberg A, Magid MA. Treatment acceleration program and the experience of the DREAM program in prevention of mother-to-child transmission of HIV. AIDS 2007;21(Suppl 4):S65-S71.

26. Kilewo C, Karlsson K, Ngarina M, et al. Prevention of mother-to-child transmission of HIV-1 through breastfeeding by treating mothers with triple antiretroviral therapy in Dar es Salaam, Tanzania: the Mitra Plus study. J Acquir Immune Defic Syndr 2009:52:406-416.

27. de Vincenzi I. Triple antiretroviral compared with zidovudine and single-dose nevirapine prophylaxis during pregnancy and breastfeeding for prevention of mother-to-child transmission of HIV-1 (Kesho Bora study): a randomised controlled trial. Lancet Infect Dis 2011;11:171-180.

28. Thomas TK, Masaba $R$, Borkowf $(B$, et al. Triple-antiretroviral prophylaxis to prevent mother-to-child HIV transmission through breastfeeding--the Kisumu Breastfeeding Study, Kenya: a clinical trial. PLoS Med 2011;8:e1001015.
29. Kuhn L, Stein Z. Infant survival, HIV infection and feeding alternatives in less developed countries. Am J Public Health 1997;87:926-931.

30. Mofenson LM. Antiretroviral drugs to prevent breastfeeding HIV transmission. Antiviral Therapy 2010;15:537-553.

31. McIntyre JA, Hopley M, Moodley D, et al. Efficacy of short-course AZT plus 3TC to reduce nevirapine resistance in the prevention of mother-to-child HIV transmission: a randomized clinical trial. PLoS Med 2009;6:e1000172.

32. Gray GE, Urban $M$, Chersich $M$, et al. A randomized trial of two postexposure prophylaxis regimens to reduce mother-to-child HIV-1 transmission in infants of untreated mothers. AIDS 2005;19:1289-1297.

33. Hoffman RM, Black V, Technau K, et al. Effects of highly active antiretroviral therapy duration and regimen on risk for mother-to-child transmission of HIV in Johannesburg, South Africa. J Acquir Immune Defic Syndr 2010:54:35-41.

34. van der Merwe K, Chersich MF, Technau K, Umurungi Y, Conradie F, Coovadia A. Integration of antiretroviral treatment within antenatal care in Gauteng Province, South Africa. J Acquir Immune Defic Syndr 2006;43:577-581.

35. Black V, Hoffman RM, Sugar CA, et al. Safety and efficacy of initiating highly active antiretroviral therapy in an integrated antenatal and HIV clinic in Johannesburg, South Africa. J Acquir Immune Defic Syndr 2008;49:276-281

36. Kuhn L, Aldrovandi GM, Sinkala M, Kankasa C, Mwiya M, Thea DM. Potential impact of new World Health Organization criteria for antiretroviral treatment for prevention of mother-to-child HIV transmission. AIDS 2010;24:1374-1371. 